Cahiers de recherches médiévales

Journal of medieval studies

$8 \mid 2001$

La protection spirituelle au Moyen Âge

\title{
Poème mondain ou œuvre morale ?
}

L'ambiguiité d'un dit de Christine de Pizan

\section{Liliane Dulac}

\section{(2) OpenEdition}

Journals

Édition électronique

URL : https://journals.openedition.org/crm/407

DOI : $10.4000 / \mathrm{crm} .407$

ISSN : 1955-2424

Éditeur

Honoré Champion

Édition imprimée

Date de publication : 15 janvier 2001

Pagination : 209-224

ISSN : 1272-9752

Référence électronique

Liliane Dulac, «Poème mondain ou œuvre morale ? », Cahiers de recherches médiévales [En ligne], 8 | 2001, mis en ligne le 13 mars 2008, consulté le 15 décembre 2022. URL : http://

journals.openedition.org/crm/407; DOI : https://doi.org/10.4000/crm.407

Ce document a été généré automatiquement le 15 décembre 2022.

Tous droits réservés 


\title{
Poème mondain ou œuvre morale?
}

\author{
L'ambiguité d'un dit de Christine de Pizan ${ }^{1}$
}

\author{
Liliane Dulac
}

1 Personne ne songerait à reconnaître dans Le Dit de Poissy ${ }^{2}$ une étape majeure dans la carrière littéraire de Christine de Pizan. Pourtant ce dit est loin d'être sans attraits, dans les parties descriptives notamment, dont la couleur, le rythme et le ton, plus personnels que d'ordinaire, débordent le cadre des conventions du genre. Cependant c'est surtout la construction surprenante, voire bizarre de ce poème qui le distingue et peut susciter l'intérêt : car cette particularité fournit l'occasion de proposer quelques réflexions qui permettront peut-être de mieux comprendre un moment important de l'évolution littéraire et morale de Christine.

2 Le Livre du Dit de Poissy, qui compte plus de 2000 vers, est daté d'avril 1400. L'œuvre appartient donc à la première partie de la carrière de Christine de Pizan, consacrée à la poésie légère et amoureuse. Le sujet en est simple, apparemment du moins : s'adressant

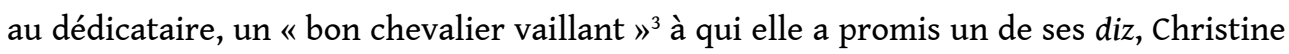
lui annonce qu'il lui sera demandé de se constituer en «juge » pour trancher le débat de deux amants. Cependant l'ensemble du poème se présente comme le récit d'une excursion de deux jours aux environs de Paris. La narratrice, Christine elle-même, se rend à Poissy par un beau matin de printemps, à cheval et accompagnée d'un groupe d'amis, des gens de la cour : elle va voir sa fille qui est religieuse dans la célèbre abbaye. Après un bref séjour à Poissy, la joyeuse compagnie repart pour Paris et pendant le retour Christine assiste au débat d'une dame et d'un écuyer, amants désespérés dont chacun se prétend le plus malheureux. Christine présente leurs récits et leurs arguments au dédicataire du poème qu'elle invite à juger le cas.

Quand Christine écrit le Dit de Poissy, elle est encore essentiellement un poète de cour. Sa carrière littéraire a commencé une dizaine d'années auparavant, peu après son veuvage. Cependant le Dit se situe à un tournant de son œuvre. Le poème est contemporain de son premier recueil des Cent Ballades, de son Epistre au dieu d'Amour, dirigée contre l'antiféminisme dont elle accuse le Roman de la Rose; on peut le rapprocher d'autres controverses courtoises qu'elle propose dans plusieurs poèmes. C'est là un thème à la mode et, bien sûr, parfaitement factice. Mais en ce tout début du 
siècle, Christine compose également l'Epistre Othea ${ }^{4}$, sa première œuvre en prose et en vers à la fois, écrite pour le duc Louis d'Orléans, le frère du roi : une œuvre allégorique complexe, qui tout en se rattachant à la littérature courtoise, est déjà un ouvrage politique, comme l'a montré Sandra Hindman ${ }^{5}$. Ce livre qui préfigure la seconde carrière de Christine de Pizan, celle de l'écrivain philosophe, connaîtra plusieurs éditions manuscrites pour des dédicataires différents.

Le travail littéraire de Christine ne peut en effet être séparé de la demande de la cour pour laquelle elle écrit. Quand ses œuvres ne sont pas directement commandées par ses protecteurs, elles doivent du moins répondre au goût de son public. Pourtant, au moment d'analyser un poème aussi marqué par les conventions que le Dit, il convient de rappeler à quel point elle saura ruser avec ces contraintes et en tirer même profit pour stimuler son invention littéraire. Si elle accepte les données de départ qu'on lui fournit, ou que l'attente des lecteurs lui impose, elle en joue assez habilement pour en déplacer, voire en inverser le sens. Avant de revenir au Dit de Poissy, je rappellerai un exemple de ce genre d'opération qui ne nous en éloignera qu'en apparence. Vers 1409, alors qu'elle a déjà écrit plusieurs grands ouvrages de philosophie morale et de politique, elle se voit commander une œuvre de poésie amoureuse. C'est prétend-elle, une amende qu'on lui impose pour avoir médit de l'amour. Cependant les Cent ballades d'amant et de dame $e^{6}$ seront loin d'être l'œuvre légère qu'on attendait peut-être d'elle : c'est une histoire tragique où la relation amoureuse se défait sans remède. Les amants en arrivent à ne plus se comprendre, chacun se plaint de voir ses sentiments méconnus et se croit victime de l'autre. Mais seule la dame en meurt. La composition de ce cycle est si subtile que le cheminement qui conduit à la catastrophe parait à la fois inéluctable et mystérieux. L'auteur laisse parler les amants, n'explique rien et ne prend pas parti ouvertement. En bien des points, l'histoire reste énigmatique, et seul est clair le malheur d'aimer.

5 Le débat que propose le Dit de Poissy est en comparaison tout à fait conventionnel et sans profondeur. Mais à considérer le poème lui-même, dont le cas à juger n'occupe qu'une partie, on s'aperçoit qu'il n'en est sans doute pas de même. Là aussi Christine nous laisse en présence d'énigmes non résolues. Elle part en effet d'une matière préexistante, un dit de Guillaume de Machaut, en conserve le thème mais en déforme à ce point les proportions que son intention devient problématique. Dans le Jugement dou roy de Behaingne ${ }^{7}$, Machaut présentait déjà le débat de deux amants dont chacun prétendait remporter la palme du malheur d'aimer sans espoir: le roi était invité à trancher le débat. Chez les deux poètes, les amants, une dame et un écuyer, sont séparés de l'objet de leur amour, l'un par un obstacle physique, l'autre par un obstacle moral qui ne laissent à tous deux aucun recours. Christine a cependant modifié les termes du problème exposé par Machaut: ce n'est plus la mort, mais un emprisonnement indéfini chez les Turcs qui prive la dame de tout espoir de revoir son ami ; quand à l'écuyer, il n'a pas été trahi par celle qu'il aime, mais seulement rebuté avec dureté, après avoir été admis à la servir, sans oser se déclarer. Considéré dans son ensemble, le Dit de Poissy est en outre organisé d'une manière toute différente des dits amoureux de Machaut, comme de ceux de Christine elle-même.

6 L'épisode du débat des deux amants n'intervient qu'au vers 1071, c'est-à-dire à plus de la moitié du poème, qui en compte 2075 ; dans le Dit dou roi de Behaingne d'une longueur analogue, le récit correspondant commence au vers 125 . Les commentateurs ont éprouvé quelque difficulté à rendre compte du partage du Dit de Poissy en deux grandes 
parties, ou plutôt deux éléments constitutifs qui semblent n'avoir guère de rapport l'un avec l'autre. "Pour situer ce débat entre deux amants malheureux [...], écrit MarieJosèphe Pinet, Christine a imaginé un voyage à Poissy où sa fille était religieuse " $^{8}$. Toutefois il est bien évident que le récit du voyage et du séjour à Poissy, qui occupe une place si importante, ne peut constituer une simple introduction destinée à situer le débat. D'ailleurs ce débat a lieu non à Poissy, mais dans un endroit indéterminé, sur la route du retour vers Paris; et il faut noter qu'il se déroule hors de la présence de la joyeuse compagnie qui entoure l'auteur-narrateur depuis le début de leur excursion. Si les deux amants infortunés appartiennent à ce groupe, la première partie du Dit ne leur donne aucun rôle, puisqu'ils ne sont individualisés comme personnages qu'au moment où commence l'épisode où ils vont s'opposer.

7 Le peu de relations entre les deux parties du poème n'a pas manqué d'opérer une sorte de disjonction entre la lecture qui a été faite de l'une et de l'autre. Plusieurs critiques, loin de ne voir qu'un hors-d'œuvre dans ce qui précède le débat des amants, ont montré beaucoup d'intérêt pour l'évocation du voyage et du séjour à Poissy. Ils en ont vanté le charme et la valeur documentaire ${ }^{9}$, tout en jugeant que la suite n'est que « pur verbiage de la littérature courtoise ». Cependant l'hétérogénéité des deux épisodes du poème pourrait être considérée comme un effet extrême d'une certaine "loi du discontinu ", caractéristique du dit comme genre. Jacqueline Cerquiglini a bien montré les multiples formes de rupture ou d'éclatement qu'on peut observer dans les dits, autour de la personne centrale de l'auteur représenté dans le texte. Dans le Dit de Poissy, il semble bien en effet que la poésie surtout descriptive du premier épisode, les paysages de printemps, les splendeurs de l'abbaye royale, et d'autre part les portraits amoureux et les argumentations subtiles de la seconde partie relèvent de codes différents et ne puissent viser à éveiller le même type d'intérêt chez le lecteur. Mais on sait que la diversité thématique ou formelle qu'on observe dans beaucoup de dits ne nuit pas à leur cohérence, qui reste forte, notamment dans les poèmes de Christine : par exemple dans le Livre des trois jugemens ${ }^{10}$ construit comme une série de débats parallèles, ou dans le Dit de la Pastoure ${ }^{11}$ qui inclut "bergerietes " et "balades». Or, précisément, il convient de souligner à quel points d'importants éléments de structure confèrent à l'ensemble du Dit de Poissy une forte unité, au moins formelle, comme l'a bien montré Barbara Altmann ${ }^{12}$ : ce sont en particulier un rigoureux emboîtement des différents récits, l'implication constante de l'auteur-narrateur, et aussi des effets de focalisation, à partir de la position du narrateur, dont nous aurons à reparler. Ces dispositifs, en rendant plus solidaires deux parties de tonalités opposées, invitent à s'interroger sur la valeur possible du contraste ainsi mis en valeur.

On ne saurait certes tenir pour établi que le Dit comporte une signification morale précise, une «leçon » à découvrir : on pourrait aussi y reconnaître une unité plus lâche, un thème à variations, comme le suggère Nicole Chareyron qui trouve aux nombreuses descriptions qu'il comporte, malgré les différences profondes qui les séparent, tous les caractères de la rêverie nostalgique, avec sa constante obsession du détail vécu ${ }^{13}$. En orientant un peu autrement la réflexion, on peut aussi songer que Christine a toujours aimé s'exprimer de manière figurée et symbolique. Son goût pour les savantes "couvertures poétiques» est trop connu pour qu'on ne s'interroge pas sur le sens caché que pourrait avoir l'architecture insolite du Dit de Poissy. La tentative faite en ce sens par Kathleen B. Kells dans un article paru en 1988 est digne d'intérêt dans la mesure où elle invite à considérer comme significative l'opposition entre les deux parties du poème qui formeraient un diptyque ${ }^{14}$. Rappelant qu'à la même époque 
Christine avait pris la défense de son sexe dans la querelle du Roman de la Rose, elle propose de voir dans le Dit de Poissy une représentation figurée de deux modes d'existence des femmes de l'aristocratie : sous le régime matriarcal du couvent, elles sont énergiques, fortes et heureuses, alors que la vie de cour, évoquée dans le débat des amants, multiplierait pour elles les risques de perdre leur réputation et leur bonheur. De façon analogue, mais avec un éclairage un peu différent, Barbara Altmann propose de reconnaître dans l'opposition entre l'épisode de Poissy et le débat des amants malheureux, une invitation à réfléchir sur les avantages de la vie conventuelle et l'expression indirecte d'une attirance personnelle pour un mode de vie que Christine adoptera beaucoup plus tard ${ }^{15}$. Cependant, avant de reprendre l'analyse des éléments qui nous paraissent les plus significatifs dans l'unité contrastée du poème, nous dirons brièvement pourquoi cette interprétation, malgré l'intérêt incontestable qu'elle présente, nous semble pouvoir être complétée pour rendre compte de l'ensemble du Dit.

9 Notons tout d'abord que dans l'hypothèse où s'y exprimerait, même confusément, la tentation de trouver un refuge dans la clôture monastique de l'abbaye royale, on pourrait trouver étonnant que la spiritualité et les activités contemplatives y tiennent si peu de place : les règles de l'ordre et les pratiques dévotes sont bien mentionnées (vv. 666-672), mais très brièvement, et sans qu'il y ait aucune rupture dans le récit. Ce qui en fait ne saurait surprendre, puisque la description du lieu comme l'évocation des heures qu'y passent la narratrice et ses amis sont entièrement présentées du point de vue tout mondain qui est le leur. Aussi est-ce la vie matérielle de l'aristocratique abbaye qui est placée au premier plan, vie luxueuse (malgré la révélation par la narratrice de certains traits d'austérité), bien protégée et bien réglée, qui telle que la découvre le groupe ne s'oppose en rien à ses goûts et à ses plaisirs ordinaires, même si les conversations sur la matière d'amour sont un instant suspendues. Cette remarque nous amène à considérer que le contraste qui au sein du poème est vraisemblablement le plus significatif n'oppose nullement la vie conventuelle et le malheur solitaire des amants, mais plutôt ce dernier au bonheur apparent de deux communautés qui vivent dans l'harmonie, le groupe des amis et celui des religieuses qui les accueillent dans leur fastueuse retraite. Notons que l'euphorie mondaine du groupe, constamment mise en valeur par la narratrice qui la partage, n'est nullement altérée par l'épisode pathétique du débat: elle se prolongera après le retour à Paris, jusqu'au dernier repas pris en commun. Nous terminerons sur une dernière remarque cette rapide discussion de l'interprétation qui met en avant le thème des deux voies qui s'offriraient aux femmes de l'aristocratie - la cour ou le couvent : elle nous paraît négliger la figure de l'écuyer malheureux, dont l'histoire pourrait pourtant résumer à elle seule l'opposition qui court tout au long du poème - nous y reviendrons -, entre la face riante de l'amour (illustrée par les assemblées mondaines où l'on converse et où l'on chante) et sa face pathétique.

Des interprétations que nous venons d'évoquer, il semble qu'on peut retenir comme hypothèse euristique l'idée d'une unité significative des deux grands développements du poème, l'excursion dans son ensemble et le débat des amants : en effet l'hypothèse inverse d'un fonctionnement totalement disjoint, totalement éclaté, ne s'accorde nullement avec ce qu'on peut observer dans les autres dits de Christine. Nous entreprendrons donc d'examiner comment se constitue dès le début du récit un réseau de motifs et de traits textuels qui ne sont pas sans rapport avec ceux qu'on peut 
observer dans le second élément constitutif du poème, l'épisode des deux amants malheureux, qui se trouve dans une position d'inclusion au sein du premier.

11 Le poème commence par le récit de la chevauchée qui mène de Paris à Poissy la narratrice et ses compagnons. Ce récit de cent cinquante vers se confond avec l'évocation de la nature en fête. On y retrouve tous les traits qui constituent traditionnellement le paysage propice au sentiment amoureux: les prés reverdis, la terre enluminée de rosée, les "florettes", les "oysillons", les alouettes et les rossignols sont autant de clichés de la poésie courtoise et des pastorales. Ce n'est qu'en poésie, par exemple, que les rossignols chantent en plein midi. Cependant, Christine a retravaillé ces motifs pour leur donner une certaine densité picturale, par exemple en multipliant les notations de couleurs.

12 Malgré leur caractère conventionnel, ces descriptions inaugurent une série qui prendra son plein développement, sous une forme plus originale, avec la représentation de l'abbaye royale, également marquée, comme nous le verrons, par l'abondance des détails matériels. Au cours de l'évocation de la chevauchée jusqu'à Poissy, le lecteur peut discerner les vues successives que découvre la joyeuse cavalcade, la Seine et ses îles, un coin de la forêt de Saint-Germain... : autant de tableaux qui se trouvent ainsi intégrés au récit. À cet égard le fait stylistique le plus important est le rappel constant $\mathrm{du}$ «nous » désignant le groupe en route pour l'abbaye, car tout, dans ces paysages, n'existe que par le regard et les impressions de la narratrice et de ses amis. L'effet de focalisation est très net par exemple dans l'évocation des bords de la Seine :

Prés de Seine venimes approchans

A lie chiere.

Si fist plus bel encor sur la riviere,

Car oisillons de plus lie maniere

Par ces ysles a haulte voix plainiere

Se deduisoient

Si liement que tous esjouïssoient

Les cuers de nous, et trop fort nous plaisoient

Arbres et prez qui partout verdissoient,

Et ces saussoies [...] (vv. 131-140)

13 Si le « je » de l'écrivain intervient en position de témoin («ce croy je ", «à mon avis », « je ne cuide mie »), le « nous » lui est souvent associé dans cette fonction comme pour mieux marquer que la narration exprime une impression collective. Ainsi dans ces vers où Christine conclut sur un mode personnel l'épisode de la chevauchée :

Si croy pour vray qu'Amours ot apresté

A cellui jour toute gaye honnesté ;

Aussi croient ceulz qui orent esté

0 moy le jour (vv. 205-208)

14 L'implication fréquente dans les descriptions du personnage collectif, dont le « je » ne se sépare pas, exprime avec insistance la joie partagée. Cette unanimité se fortifie de toutes les impressions du voyage, à commencer par les sensations les plus simples, celle par exemple d'un vent léger qui soulève le manteau des cavaliers :

\footnotetext{
Alors fichié s'est entre nous et mis

Un ventelez

Doulz et plaisant, qui noz cours mantellez

Nous soubslevoit souefs et freschelés,

C'est zephirus qui boutons novellez

Fait espanir

Et ces belles doulcetes fleurs venir
} 
Et aux amans donne maint souvenir

De leurs amours ; (vv. 151-159) alentours semble devoir son charme à l'objet même qui est donné à voir dans ces quelques sept cents vers. Marie Josèphe Pinet écrit de manière très significative que «la poésie de ce dit est dans la vie d'une abbaye d'il y a cinq siècles " ${ }^{16}:$ comme s'il avait suffi que Christine la décrive exactement pour faire œuvre poétique. Plusieurs commentateurs vantent également la valeur documentaire de cet épisode, dont le langage semble n'être que pure transparence, comme une sorte de fenêtre ouverte sur un spectacle charmant. Une telle impression est sans doute renforcée par la relative originalité de ce morceau, qui porte moins que d'autres la marque de conventions littéraires. En réalité ce long passage est fort comp1exe et son efficacité poétique est moins due à la beauté du modèle qu'à un effet de l'art. La perspective narrative adoptée au début du Dit est pour l'essentiel maintenue: les descriptions sont étroitement intégrées au récit de la journée passée à Poissy et elles interviennent aux différentes étapes de la visite ou à l'occasion des scènes qui réunissent la compagnie venue de Paris et les religieuses. Ce sont souvent de brèves notations insérées dans la trame narrative, comme cette peinture des apprêts d'un déjeuner :

[la prieure] ... nous fist mener

En une chambre

Belle, plaisant, la ou ${ }^{17}$ ot fait estendre

Nappes flairans blanches et tapis tendre;

Vins, viandes aportent sans attendre

A grant largece

En vaissiaulz d'or et d'argent par noblece ; (vv.355-361)

Brèves ou plus développées, les descriptions étonnent par la surabondance des détails : l'espace de la représentation est saturé, comme celui d'une tapisserie flamande ou de certaines miniatures, pour un effet qui est poétique et symbolique. En effet, l'ensemble du décor, les bâtiments et leurs ornements, les fontaines, les viviers, les jardins, les arbres et les fruits, le parc et les animaux, mais aussi les costumes, la vaisselle, les mets, les boissons, tous ces éléments matériels, si divers qu'ils soient, concourent à peindre un univers de beauté et d'harmonie, d'ordre et de richesse. On croira volontiers que 
Christine décrit exactement ; mais elle offre surtout à l'imagination un espace de rêve, d'une perfection et d'une plénitude telles que ne peut subsister aucune faille où se glisseraient le défaut, la laideur, le danger.

S'agit-il d'une vision idéalisée de l'abbaye? Sans doute. Mais il faut observer que l'hôtellerie de Bourbon toute voisine, où le groupe sera hébergé, est dépeinte en termes analogues :

Mais on feroit bien une grant légende

Du long parler, de la chiere très grande

Qu'on nous ot fait et du lieu ou lavande

Croist et rosiers

A grant foison sans façon de closiers,

C'est es jardins ou a maint cerisiers (vv. 609-614)

Bien que plus rapidement évoqué, ce lieu profane n'est lui aussi que beauté et délices, et telle l'abbaye, un espace à la fois clos, donc protégé, et accueillant : aux « haulz murs bien clos » du jardin des dominicaines., aux « grans portes, fortes et bien barrées » de leur couvent (v. 562 et v. 597) répond à l'hôtellerie une

[...] cloison

Fort et belle pour oster l'achoison

Des maulx qu'on fait au monde a grant foison. (vv. 616-618)

La relation entre les deux lieux est d'ailleurs étroite puisque c'est du couvent que viennent, pour les repas pris à l'hôtellerie, le bon vin

De Saint Porçain,

En poz dorez, largement et a plain. (vv. 776-777)

et aussi des desserts,

Bonnes goieres

Bien sucrées, bien faittes et legieres,-

Pomes, poires de diverses manieres. (vv. 788-790)

21 Là encore la fonction des détails, qui peuvent paraitre surabondants, est de signifier que chacun des instants évoqués est en lui-même parfait. Ainsi le moment du départ, celui où la compagnie va reprendre la route de Paris, est décrit de manière étonnamment minutieuse :

Primes congié des dames, puis montames

Sur haquenées

Grosses, belles, gentement ordennées,

Qui ains partir furent bien desjunées ; (vv.891-894)

D'autres éléments du récit tendent à donner la même impression de plénitude, notamment la temporalité précise et continue de tout l'épisode. Des indications nombreuses marquent clairement les étapes de la visite et le rythme de la vie conventuelle. La journée est ponctuée par les collations : les déjeuners, un dîner et un souper, pris à l'abbaye ou à l'auberge, avec des intervalles souvent indiqués :

Ne fusmes mie

Après disner, je croy heure et demie

Quant celle, qui est maistresse et amie

De ma fille, nous manda ; (vv. 628-631)

Les moments et les actions forment une succession ininterrompue parfois détaillée à l'extrême, exactement comme les descriptions sur le plan spatial. Ainsi le poème donne l'idée d'un temps entièrement exprimé par le récit :

Et puis lavames

Après disner noz mains et nous levames, 
Et tout en piez une piece parlames,

Puis reposer un petit nous alames [...] (vv. 620-623)

La durée est d'autant mieux ressentie comme homogène et sans faille, sans vide, qu'elle garde toujours un caractère collectif: le bonheur et les plaisirs de cette journée appartiennent également à tous, ainsi que le soulignent plusieurs scènes de groupes; ce sont des conversations dévotes avec les religieuses, des danses et des chansons à l'hôtellerie, un débat sur les qualités des meilleurs chevaliers, des chants et des jeux au bord de la Seine, à la tombée de la nuit... Aucun personnage de la joyeuse compagnie ne s'individualise, sinon pour exprimer le vœu de tous, comme cette «belle damoiselle jolie » qui propose de danser la carole (une sorte de ronde, qui en elle même pourrait symboliser l'unité du groupe) (v. 728). Seule exception, le moment où la narratrice, dans son rôle de mère, s'afflige de quitter sa fille : significativement, cet instant unique où un personnage se détache est aussi le seul où la tristesse affleure. Mais le chagrin est aussitôt chassé par l'intervention empressée des amis :

Et a parler pristrent d'aultre dittié

Pour m'oublier

Et moy tollir a malencolier [...] (vv. 711-713)

Bien sûr, à l'auberge et au prieuré les plaisirs de la société ne sont pas identiques. Dans les jardins de l'abbaye, au bord des fontaines, dames et chevaliers ne se livrent ni aux plaisirs de la danse, ni aux entretiens sur la matière d'amour :

N'y parlames d'amours ne ne dançames,

Ains enquismes tout et leur demandames

De leur ordre les points, et n'y pensames

Decepcion,

La n'ot parlé fors de devocion [...] (vv. 665-669)

Mais la différence entre les moments passés à l'auberge et ceux passés à l'abbaye est de pure convenance. Le tour négatif donné à ce passage souligne combien le bonheur qui s'exprime dans l'évocation de ces lieux est en fin de compte semblable. Le regard posé sur le monastère est toujours celui de la compagnie venue de Paris : elle y voit « un trés doulz paradis » (v. 557), en l'associant le plus souvent à des plaisirs qui sont surtout des plaisirs mondains. On en trouvera une preuve dans une indication discrètement érotique à propos du couvent et des dominicaines qui l'habitent:

Mais bien estre y voulsissions toutjour,

Car aux hommes trop plaisoit la doulçour

De ces dames qui de moult simple atour

Furent voillées ;

Si ne furent ne noires ne hallées,

Mais comme lis blanches et potellées. (vv. 649-654)

27 De ce point de vue, les religieuses si séduisantes et plus « freschetes que rose » peuvent être comparées à l'agréable compagnie que le groupe retrouve à l'hôtellerie :

Adonc de la dance nous departon,

Ou il avoit maint joli valeton,

Mainte belle pucelle a doulz menton,

Mignote et gente [...] (vv. 753-756)

La description de l'abbaye est donc dans le prolongement du début du poème, le récit de la joyeuse chevauchée depuis Paris. L'unité d'atmosphère du voyage et du séjour à Poissy est d'ailleurs mise en valeur par quelques traits champêtres et bucoliques qui, vers la fin de la journée, rappellent les paysages traversés le matin : 
Tout en parlant, par dessus le pavé

Somes alez jouer tant que trouvé

Avons les champs,

Ou grant déduit prenions d'oïr les chans

Des rossignolz quant fumes approchans

Des ysletes sur Seine [...] (vv. 870-875)

Ainsi jusqu'au moment où intervient le débat des amants malheureux, tous les détails contribuent à représenter la plénitude du bonheur partagé par tous, sans l'ombre d'une inquiétude ou d'une souffrance.

Je ne m'attacherai pas à analyser le débat des amants qui disputent pour savoir qui des deux est le plus malheureux. Les éléments qui composent l'épisode sont ceux qu'on retrouve dans la plupart des débats de ce genre : un récit, un portrait détaillé de l'être aimé, une longue et subtile casuistique qui constitue le débat proprement dit. Notons pourtant que l'évocation des charmes physiques est d'une précision remarquable, et elle peut surprendre quand il s'agit de la beauté virile de l'amant, telle que la perçoit la dame qui s'en souvient avec nostalgie et volupté :

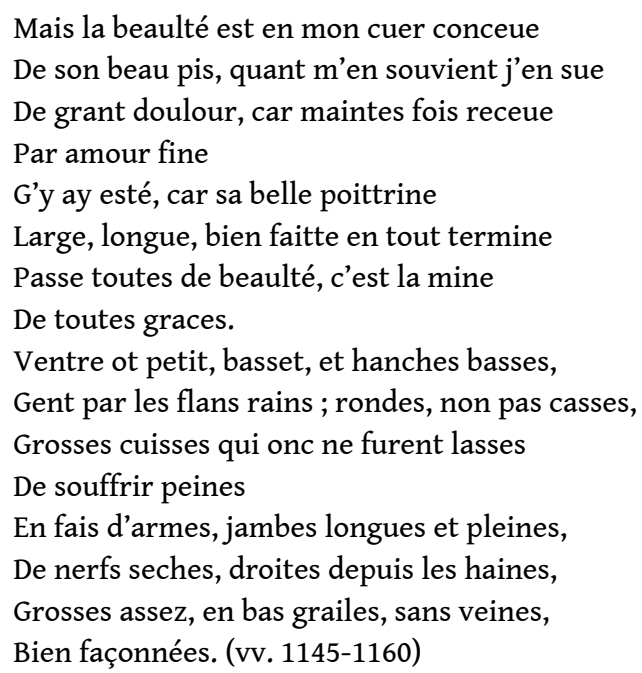

Un critique de la fin du XIX ${ }^{\mathrm{e}}$ siècle s'est scandalisé de ce langage ${ }^{18}$ : c'est sans doute dans cette seule occasion qu'on a suggéré que Christine de Pizan pouvait être un écrivain obscène. Mais la sensualité de ces descriptions, peut-être poussée un peu plus loin que la tradition ne l'exige, n'est pas un élément négligeable dans le poème. Elle rejoint d'autres traits nombreux qui concourent à vanter les plaisirs de la vie.

Cependant, considéré dans son ensemble, cet épisode du débat des amants est en parfaite opposition avec le récit du voyage et du séjour à Poissy. Tout en effet semble les séparer. La première partie donne l'image d'un bonheur parfait, solidement fondé sur des réalités délicieusement présentes; la seconde évoque l'être aimé à jamais inaccessible : ces portraits sensuels sont donnés en majeure partie au passé. À la durée calme et bien rythmée de la vie heureuse s'oppose une attente indéfinie et, dans le cas de la dame affligée, les tourments d'une âme déchirée entre la rage des vains regrets et les projets désespérés. Aux épisodes qui expriment la joie collective font place une scène à trois, et l'aveu de souffrances solitaires. Cependant un contraste aussi accentué suggère déjà une sorte de relation paradoxale que la dame indique pour l'avoir éprouvée dans le moment même, car au milieu d'un groupe joyeux, la souffrance personnelle est plus amère : 
Et pourtant se bonne chiere en commun

Je fais, certes mon cuer n'a bien nesun,

Et moult souvent plorer devant chascun

Il me convient

Quant grant desir trop fort sur moy survient,

Car sans cesser de cellui me souvient

Qui a mon cuer, qu'en prison on retient

Si durement,

Et quant plus suis en grant esbatement

Lors me souvient plus de son grief tourment

Qui ma joye rabat trop durement. (vv. 1349-1359)

Ce thème est proche de celui que Christine a développé plusieurs fois sur un mode personnel en évoquant son veuvage et sa solitude ${ }^{19}$ :

De triste cuer chanter joyeusement

Et rire en dueil, c'est chose fort a faire,

De son penser moustrer tout le contraire,

N'yssir doulz ris de doulent sentement.

Ainsi me faut faire communement (Rondeau XI)

Dans l'organisation du texte, certains rapports entre les deux éléments du Dit sont perceptibles. Comme dans beaucoup de poèmes de ce genre, le "je» du narrateur constitue un premier lien qui peut certes réunir de façon formelle des éléments disparates. Mais le « je » du Dit de Poissy est loin d'être sans consistance, et, entre autres fonctions, il faut observer celle, qu'il assume constamment, de l'auteur présent dans son œuvre: il est celui qui s'adresse au dédicataire du poème qu'il signera de l'anagramme de son nom («creintis», v. 2075); il est le clerc qui a promis à ce «bon chevalier » un «beau dit » (v. 857) et s'est trouvé chargé d'exposer en "briefs termes » le cas à juger (v. 2047); en cela il est aussi et surtout un témoin, véridique par définition. En traduisant par l'écriture les paroles des amants malheureux, la poétesse atteste leurs souffrances dont elle enregistre les signes indubitables. Il en est ainsi de l'écuyer dont elle requiert l'assistance, parce qu'elle le devine en proie au mal d'amour :

Mais souspirant

Aloit souvent, bien croy qu'en desirant

Avoit maint mal. (vv. 924-926)

Ce « je » de l'auteur a d'autant plus de présence qu'il n'est pas un spectateur passif, mais l'instigateur du débat qui va s'engager : c'est lui qui réunit les deux amants et provoque leurs confidences. Ce rôle de témoin actif existe également, dans la première partie, mais de manière différente: la narratrice proclame en effet fréquemment la vérité de ses descriptions, ou leur insuffisance, quand elle veut donner à imaginer une réalité qui dépasse les possibilités du langage. Cet auteur qui ne s'efface jamais derrière les êtres et les choses qu'il met en écriture apparaît donc comme responsable d'un texte qu'on ne peut croire livré à l'aventure et formé d'une succession capricieuse d'éléments divers. Il n'est pas inutile de revenir ici sur un trait qui constitue un autre marque de cette volonté organisatrice: le débat des deux amants constitue un épisode soigneusement enchâssé dans le récit principal, celui de l'excursion de Poissy. Il est introduit aux vers 903-904 par un cliché dont apparaissent plusieurs variantes au cours du poème : la nature printanière réveille les souvenirs d'amour ${ }^{20}$. Mais il faut surtout noter que les trois personnages qui, lors de cet épisode, s'écartent de la noble compagnie - depuis le commencement héros principal de l'histoire - la rejoignent à la fin : 
Et de noz gens, dont estions departies,

Nous approchames

Et liement ensemble chevauchames

Tant que chieux moy a Paris arrivames,

Ou a grant joye et a festes disnames. (vv. 2055-2059)

Ainsi se trouve brièvement rétabli le climat joyeux de la première partie, à l'occasion
d'un repas pris en commun qui sera le dernier d'une longue série. Les tristes récits et
les plaintes des deux amants semblent effacés. Il n'en reste rien.

et à la fin de l'œuvre comme le sujet même du Dit, la continuité du récit premier (l'excursion à Poissy) le fait apparaître en réalité comme une digression. Parce qu'il est constitué en partie de récits au second degré, l'épisode forme une longue incise d'une tonalité toute différente. Il y a donc là une sorte de contradiction, mais elle n'est qu'apparente et nous conduit à constater un trait qui nous paraît essentiel dans l'organisation du texte. Il existe en effet dans le récit du voyage et du séjour à Poissy, et d'autre part dans le débat des amants, des éléments analogues, quoique de valeur inverse. Avant que n'intervienne la controverse sur le malheur d'aimer, d'autres discussions sur les matières d'amour sont mentionnées, beaucoup plus brièvement il est vrai. Ainsi pendant la chevauchée vers Poissy, c'est-à-dire à une place symétriquement opposée à celle du grand débat :

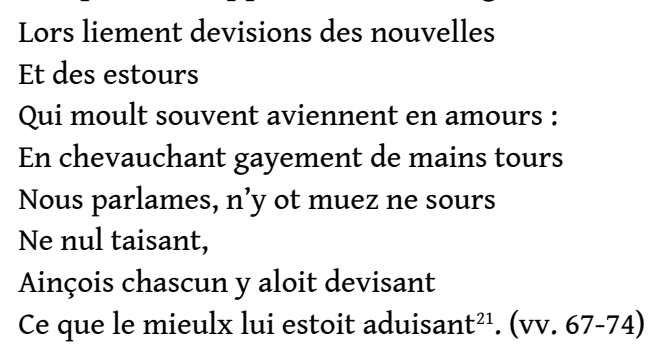

Une autre conversation sur l'amour est encore mentionnée à la fin de ce voyage :

Car d'esbatre ne cessames tousjour

Rire et jouer, et chanter sans sejour,

Ou deviser d'aucun parti d'amour. (vv. 209-211)

Plus tard, au cours d'un autre entretien qui porte sur les meilleurs chevaliers du royaume (vv. 793-861) et fournit l'occasion d'un éloge du dédicataire, on discute à nouveau d'amour et le « bon chevalier » auquel est dédié le poème est alors jugé « vray fin amant» (v. 826). Une autre référence à de telles discussions marque bien leur véritable statut. Nous avons déjà mentionné cette scène qui réunit les visiteurs et les religieuses dans le jardin du couvent et que la narratrice introduit par cette indication négative :

La nous sismes et de choses mondaines

Pou devisames,

N’y parlames d'amours ne ne dançames (vv. 663-665)

Cette précision est utile parce que les conversations sur le thème d'amour sont, parmi les plaisirs de la société, les plus habituels, comme les chants et les danses. On ne peut se réunir en compagnie sans parler d'amour et discuter de quelques cas. La grande controverse des deux amants infortunés relève de la même pratique sociale ou de la même convention littéraire. Et pourtant elle se situe à plusieurs égards à l'opposé de ces jeux aimables pratiqués par les dames et écuyers au cours de leur joyeuse excursion. Réduit aux deux protagonistes et à leur unique témoin, l'épisode révèle des 
souffrances dissimulées en société. En outre le débat, ainsi pratiqué sur une scène réduite, est en même temps démesurément amplifié : au lieu d'une dizaine de vers, il en occupera mille. Ce brusque changement d'échelle s'accompagne d'une inversion de signe : comme si le jeu d'amour, naguère si gai, se muait en vrai tourment. C'est une métamorphose dont peut faire l'expérience l'observateur qui parvient à percer la surface des plaisirs de cour, mais surtout qu'éprouve en lui-même l'amant qui se laisse d'abord aller au plaisir d'aimer, avant que ce bonheur ne se transforme en désespoir, comme le confesse le jeune écuyer :

Ainsi devins adonc d'amours acointe

Et me plut bien au de premier la pointe

Qui m'a depuis esté d'amertume ointe

Diverse et dure. (vv. 1633-1636)

41 Une autre pratique mondaine du même ordre est l'objet d'un renversement analogue au cours du Dit. De joyeuses assemblées sont évoquées deux fois dans la première partie et une fois au moins dans la seconde. Pendant le séjour à Poissy, la compagnie passe quelques heures à danser la carole et à chanter à l'auberge, plaisir intensément partagé par tous, ainsi qu'il est de règle dans cet épisode :

Et de renc puis

Chascun chanta tant qu'il fu près de nuys,

Car le dancier ne tournoit a anuys

A nul qu'y fust. (vv. 748-751)

Plus tard intervient une scène tout aussi enjouée au bord de la Seine, tandis que chantent les rossignols (vv. 870-889). Dans l'épisode des deux amants, Christine de Pizan décrit également une "assemblée ", non plus en dix, mais en cent vingt vers (vv. 1421-1461), car elle marque le début d'un amour malheureux :

En ce jardin avoit une assemblée

Belle, plaisant, ou joye estoit doublée [...] (vv. 1429-1430)

Mais à cette occasion le danger de divertissements si aimables et séduisants est nettement indiqué :

Car onc ne vi de joye tel largece

Et en ce lieu ot mainte grant maistrece

Et mainte autre parée de noblece

Et maint jolis

Gay chevalier, car de la fleur de lis

Noble et royal, ou lieu plain de delis

Avoit aucuns et d'aultres si polis

Que ce sembloient

Dieux, deesses, qui ou lieu s'assembloient,

Dont l'un a l'autre les cuers s'entr'embloient,

Moult soubtilment et du mal s'affubloient

Qui a grant joye

Est commencié et puis en griefs plours noye. (vv. 1441-1453)

C'est un lieu commun, bien sûr, développé en termes presque identiques dans le Debat de deux amans (v. 122), mais il est ici fort important et d'une valeur quasi stratégique, puisqu'il rend probablement compte de la structure même du Dit de Poissy. La joie mondaine si riche en agréments, si sûre et bien ordonnée, semblait naguère s'accorder avec la douce quiétude du couvent. Mais on découvre soudain comme par l'effet d'un miroir grossissant qu'elle peut masquer le désespoir et l'angoisse sans remèdes.

Le contraste est trop marqué, trop précisément agencé pour qu'on ne songe pas à y reconnaître un effet concerté. Ne faut-il pas voir dans l'opposition des deux parties du 
poème un montage qui soudain fait éclater les apparences les plus rassurantes ? C'est un «tres doulz paradis » (v. 557) que représente l'abbaye royale de Poissy, si riche, si calme, à la vie si douce et bien réglée : «luxe, calme et volupté » dirait un autre poète. Car si la discipline conventuelle, son austérité même sont parfois évoquées (vv. 314-323), elles signifient dans ce contexte moins la contrainte et la rigueur que la régularité paisible d'une existence bien ordonnée, dans un lieu splendide ; les gens de la cour, venus de Paris avec la narratrice, semblent s'y trouver à l'aise, comme s'ils partagaient le même bonheur, comme si leurs jeux mondains étaient aussi purs et innocents que la vie contemplative des dominicaines. Cependant cette surface aimable et riante est comme crevée par un débat dramatique. Il est certain qu'un tel montage suggère beaucoup; mais on peut douter que sa signification soit précisément déterminée. Peut-être ne faut-il y voir que le symbole transparent des deux faces de l'amour : l'une sourit, l'autre pleure ? Peut-être l'auteur du poème n'a-t-il voulu rien de plus qu'associer dans "son beau dit», pour le plaisir du lecteur, deux tonalités opposées? Cependant, comme nous l'avons rappelé en commençant, Christine sera durablement tentée par une dénonciation des pièges de l'amour courtois. En transformant la donnée fournie par Guillaume de Machaut, elle semble avoir pris ses distances par rapport à un thème obligé, le débat d'amour. Il semble en effet que les deux grands éléments constitutifs du poème, en jouant l'un par rapport à l'autre, se démasquent mutuellement : la joie mondaine peut être trompeuse; et d'autre part, les débats, les «devis » d'amour qu'on y affectionne, paraissent singulièrement factices, qu'on les confronte à des souffrances véritables, comme celles qu'atteste la narratrice, ou au contraire aux bonheurs les plus simples de la vie aristocratique, ceux que peuvent connaître une communauté paisible, comme celle de Poissy, ou une joyeuse troupe d'amis partis en excursion. C'est cette dernière image que propose une miniature du Dit de Poissy, dans la collection manuscrite offerte à Isabeau de Bavière ${ }^{22}$. Nous avons vu que le poème lui-même se termine par une scène de la même veine : un joyeux repas pris en commun chez la narratrice, de retour à Paris. Cet épilogue, en ramenant la joie collective, semble suggérer en tout cas que, s'il n'y a peut-être pas d'amour heureux, le bonheur est à portée d'une sagesse souriante. La boucle se referme. Faut-il s'en tenir à cette conclusion? Il semble que la poétesse qui a tenu la plume pour exposer le cas des amants malheureux ait songé a une leçon que son Dit pourrait enseigner. Mais quelle est-elle au juste? Que l'amour est un danger que masquent les loisirs charmants de la vie de cour? ou bien que le bonheur ne peut résider que dans les plaisirs largement partagés, dans l'ordre et le jeu réglé de la vie, à l'image des deux communautés réunies à Poissy pour un jour, alors que l'amour, qui sépare et isole, ne peut être que source d'infortunes?

\section{NOTES}

1. Cette étude développe certains aspects d'un article publié : Liliane Dulac, «Le Livre du dit de Poissy de Christine de Pizan, poème éclaté ou montage signifiant ", Écrire pour Dire, Études sur le dit médiéval, sous la direction de Bernard Ribémont, Paris, Klincksieck, 1990, pp. 9-28. 
2. Christine de Pizan, Le Livre du dit de Poissy, dans Euvres poétiques, éd. Maurice Roy, Paris, Firmin Didot, II (1891, reprinted New-York, Johnson 1965), pp. 159-222. Nous utilisons également la belle édition donnée par Barbara K. Altmann, dans The Love debate poems of Christine de Pizan, University Press of Florida, 1998, pp. 202-274.

3. À propos du dédicataire, voir les notes du Dit de Poissy dans l'édition de B. Altmann, p. 264, ainsi que Charity Cannon Willard, Christine de Pizan: Her life and works, New York, Persea Books, 1984, pp. 64-65.

4. Christine de Pizan, L'Epistre Othea, éd. Gabriella Parussa, Genève, Droz, 1999.

5. Sandra Hindman, Christine de Pizan's "Epistre Othéa ": Painting and Politics at the Court of Charles VI, Toronto, Pontifical Institute of Mediaeval Studies, 1986.

6. Christine de Pizan, Cent Balades d'Amant et de Dame, éd. Jacqueline Cerquiglini, Paris, U.G.E, 1982 (10-18, $\left.n^{\circ} 1529\right)$.

7. Guillaume de Machaut, Le Jugement dou roy de Behaingne, dans œuvres de Guillaume de Machaut publiées par Ernest Hoepffner, Paris, Firmin Didot, 1908, I, pp. 57-135. Jacqueline Cerquiglini, « Le clerc et l'écriture : le Voir Dit de Guillaume de Machaut et la définition du Dit », Literatur in der Gesellschaft des Spätmittelalters, Heidelberg, Carl Winter, Universitätsverlag, 1980, pp.151-168. Johanna Catharina Schilperoort, Guillaume de Machaut et Christine de Pisan, étude comparative, 'SGravenhage, H. P. de Swart, 1936.

8. Marie-Josèphe Pinet, Christine de Pisan, 1364-1430: étude biographique et littéraire, Paris, Champion, 1927, pp. 56-59.

9. Voir Charity Cannon Willard, « The Dominican Abbey of Poissy in 1400 », Christine de Pizan 2000. Studies on Christine de Pizan in honour of Angus J. Kennedy, ed. John Campbell and Nadia Margolis, Amsterdam (Atlanta), Rodopi, 2000, pp. 209-218, et du même auteur, Christine de Pizan, op. cit., p. 43.

10. Le Livre des trois jugemens, éd. M. Roy, II, pp. 111-157.

11. Le Dit de la Pastoure, éd. M. Roy, II, pp. 223-294.

12. Barbara K. Altmann, «Diversity and coherence in Christine de Pizan's Dit de Poissy », French Forum, vol. 12, n³, sept. 1987, pp. 261-271, notamment pp. 263-266. Voir aussi du même auteur, " «Trop peu en sçay » : the reluctant narrator in Christine de Pizan's works on love », Chaucer's French contempories. The poetry/poetics of self tradition, ed. R. Barton Palmer, New York, AMS Press, 1999, pp. 217-249, notamment p. 228 sq.

13. Nicole Chareyron, «Trois états du sentiment de l'irréversible et de la nostalgie dans le dit de Poissy de Christine de Pizan", Contexts and continuities: Proceedings of the IVth international colloquium on Christine de Pizan (Glasgow, 21-27 july 2000), ed. Angus J. Kennedy in collaboration with Rosalind Brown-Grant, James L. Laidlaw \& Catherine Müller, Glasgow University Press, 2002, 3 vol., à paraître.

14. Kathleen E. Kells, «Christine de Pizan's Le Dit de Poissy: an exploration of an alternate lifestyle for aristocratic women in fifteenth century France », Fifteenth Century Studies, 14, 1988, pp. 103-119.

15. Barbara K. Altmann, art. cit. Voir aussi, du même auteur, l'introduction au Dit dans l'édition citée, pp. 203-205.

16. Marie-Josèphe Pinet, op. cit., pp. 253-254. À propos du couvent de Poissy et des religieuses au Moyen Âge, on lira avec profit : S. Moreau-Rendu, Le Prieuré royal de Saint-Louis de Poissy, Colmar, Imprimerie Alsatia, et Michel Parisse, Les Nonnes au Moyen Âge, Le Puy, Christine Bonneton Éditeur, 1983.

17. Nous corrigeons ce dernier mot d'après l'édition de B. Altmann, éd. cit., p. 214.

18. E. M. D. Robineau, Christine de Pisan: sa vie et ses œuvres, Saint-Omer, Fleury-Lemaire, 1882, p. 66 : «[...] la dame et le chevalier dans les descriptions successives qu'ils nous font des vertus et des charmes corporels de l'objet de leur affection, sont d'un réalisme qui va jusqu'à l'indiscrétion la plus choquante ». 
19. Cuvres poétiques, éd. M. Roy, I, p. 153.

20. Roger Dragonetti, La Technique poétique des trouvères dans la chanson courtoise, Genève, Slatkine Reprints, 1979, p. 169.

21. Nous corrigeons ce dernier mot d'après l'édition de B. Altmann, éd. cit. p. 207.

22. Londres, British Library 4431 , fol. $81^{\mathrm{r}}$.

\section{AUTEUR}

LILIANE DULAC

Université Paul-Valéry, Montpellier 